\title{
ON THE DEGREES OF PROJECTIVE REPRESENTATIONS
}

\author{
by R. J. HIGGS
}

(Received 26 June, 1986)

All representations and characters studied in this paper are taken over the complex numbers, and all groups considered are finite. For basic definitions concerning projective representations see [1].

If $G$ is a group and $\alpha$ is a cocycle of $G$ we denote by $\operatorname{Proj}(G, \alpha)=\left\{\xi_{1}, \ldots, \xi_{t}\right\}$ the set of irreducible projective characters of $G$ with cocycle $\alpha$, where of course $t$ is the number of $\alpha$-regular conjugacy classes of $G ; \xi_{i}(1)$ is called the degree of $\xi_{i}$. Also as normal, $M(G)$ will denote the Schur multiplier of $G,[\alpha]$ the cohomology class of $\alpha$, and [1] the cohomology class of the trivial cocycle of $G$.

Our main result exactly describes the greatest common divisor of the degrees of $\operatorname{Proj}(G, \alpha)$.

Main Theorem. Let $p_{1}, \ldots, p_{n}$ be the prime divisors of $|G|$, with $P_{1}, \ldots, P_{n}$ corresponding Sylow $p_{i}$-subgroups of $G$. Let $M_{i}$ be a subgroup of $P_{i}$ of minimal index such that $\left[\alpha_{\left.M_{i}\right]}\right]=[1]$. Then the greatest common divisor of the degrees of $\operatorname{Proj}(G, \alpha)$ is equal to $\prod_{i=1}^{n}\left[P_{i}: M_{i}\right]$.

We start by defining

$$
s(G, \alpha)=\min \left\{\xi_{i}(1): 1 \leq i \leq t\right\}
$$

and

$$
c(G, \alpha)=\text { g.c.d. }\left\{\xi_{i}(1): 1 \leq i \leq t\right\} .
$$

It is obvious that if $[\alpha]=[1]$ then $c(G, \alpha)=s(G, \alpha)=1$. Consequently we are only really interested in non-trivial cocycles of $G$.

We now quote the following well-known result.

Lemma 1. Let $\alpha$ be a cocycle of $G$ with $o([\alpha])=e$ in $M(G)$. Then

(i) $e \mid c(G, \alpha)$;

(ii) if $p$ is a prime number such that $p \mid c(G, \alpha)$ then $p \mid e$.

We note here that it is not true in general that $c(G, \alpha)=e$, or indeed that, if some integer $m$ divides $c(G, \alpha)$, then $m \mid e$; for from [2] there exists a cocycle $\alpha$ of $G=2^{4}$ with $o([\alpha])=2$ but $c(G, \alpha)=4$.

We now show that to analyse $c(G, \alpha)$ we should consider the prime divisors of $o([\alpha])$ and $s\left(P, \alpha_{p}\right)$ for the corresponding Sylow subgroups, $P$, of $G$.

Proposition 1. Let $c=c(G, \alpha)$; then the pth part of $c, c_{p}$, is equal to $s\left(P, \alpha_{P}\right)$ for $P a$ Sylow p-subgroup of $G$.

Glasgow Math. J. 30 (1988) 133-135. 
Proof. Let $P \in \operatorname{Syl}_{p}(G)$ and $\operatorname{Proj}\left(P, \alpha_{P}\right)=\left\{\gamma_{1}, \ldots, \gamma_{r}\right\}$.

Now let $\xi \in \operatorname{Proj}(G, \alpha)$ such that $(\xi(1))_{p}=c_{p}$; then $\xi_{P}=\sum_{j=1}^{r} b_{j} \gamma_{j}$, where the $b_{j}$ are non-negative integers so that

$$
c_{p}=s\left(P, \alpha_{P}\right)\left(\sum_{j=1}^{r} b_{j} \frac{\gamma_{j}(1)}{s\left(P, \alpha_{P}\right)}\right)_{p}
$$

and hence $s\left(P, \alpha_{P}\right) \mid c_{p}$.

On the other hand let $\gamma \in \operatorname{Proj}\left(P, \alpha_{P}\right)$ be such that $\gamma(1)=s\left(P, \alpha_{P}\right)$. Then $\gamma^{G}=\sum_{i=1}^{t} a_{i} \xi_{i}$ for some non-negative integers $a_{i}$, and so comparing the $p$ th parts of the degrees we obtain

$$
s\left(P, \alpha_{P}\right)=c_{p}\left(\sum_{i=1}^{t} a_{i} \frac{\xi_{i}(1)}{c}\right)_{p}
$$

and hence $c_{p} \mid s\left(P, \alpha_{P}\right)$.

We are thus left with the task of describing $s\left(P, \alpha_{P}\right)=c\left(P, \alpha_{P}\right)$ for $P \in \operatorname{Syl}_{p}(G)$. However, we shall actually consider a more general situation than this. Recall that $\xi \in \operatorname{Proj}(G, \alpha)$ is called monomial if it is induced from a projective character of degree 1 of a subgroup, and $G$ is said to be a PM-group if all its irreducible projective characters are monomial.

Proposition 2. Let $M$ be a subgroup of $G$ of minimal index such that $\left[\alpha_{M}\right]=[1]$; then

(i) $s(G, \alpha) \leq[G: M]$ and $c(G, \alpha) \mid[G: M]$;

(ii) if $c(G, \alpha)=[G: M]$, then $c(G, \alpha)=s(G, \alpha)$;

(iii) $s(G, \alpha)=[G: M]$ if and only if there exists a monomial character $\xi \in \operatorname{Proj}(G, \alpha)$ with $\xi(1)=s(G, \alpha)$.

Proof. Let $\xi^{\prime} \in \operatorname{Proj}(G, \alpha)$ such that $\xi^{\prime}(1)=s(G, \alpha)$, and $\lambda \in \operatorname{Proj}\left(M, \alpha_{M}\right)$ with $\lambda(1)=1 ;$ then $\lambda^{G}=\sum_{i=1}^{t} a_{i} \xi_{i}$, for some non-negative integers $a_{i}$, and so

$$
\lambda^{G}(1)=[G: M]=c(G, \alpha)\left(\sum_{i=1}^{t} a_{i} \frac{\xi_{i}(1)}{c(G, \alpha)}\right) \geq \xi^{\prime}(1)
$$

proving (i). Since $c(G, \alpha) \mid s(G, \alpha)$ we have that (ii) is immediate from (i).

Now suppose that equality holds in (1); then we must have that $\lambda^{G}$ is irreducible. Conversely if $\xi \in \operatorname{Proj}(G, \alpha)$ is monomial and $\xi(1)=s(G, \alpha)$, then by definition there exists a subgroup $L$ of $G$ and $\mu \in \operatorname{Proj}\left(L, \alpha_{L}\right)$ with $\mu(1)=1$ such that $\mu^{G}=\xi$; obviously then $\left[\alpha_{L}\right]=[1]$ from Lemma $1(\mathrm{i})$. Also $[G: L]=s(G, \alpha) \leq[G: M]$ by (i), and hence by hypothesis $[G: L]=[G: M]$.

Of course equality in Proposition 2(iii) does occur when $G$ is a $P M$-group and in particular when $G$ is supersolvable $([1,(6.5 .11)])$. However if $G=A_{4}, o([\alpha])=2$, then 
$s(G, \alpha)=c(G, \alpha)=2$; but $A_{4}$ has no subgroup of index 2 , so that equality does not always hold.

The proof of the main theorem is now yielded by the above remarks in conjunction with Propositions 1 and 2.

We mention just three applications of the above results.

Corollary 1. Let $L$ be a cyclic subgroup of $G$; then $s(G, \alpha) \leq[G: L]$ and $c(G, \alpha) \mid[G: L]$ for all cocycles $\alpha$ of $G$.

Proof. Since $L$ is cyclic $M(L)$ is trivial and hence [ $\left.\alpha_{L}\right]=[1]$ for all cocycles $\alpha$ of $G$; thus the result is immediate from Proposition 2(i).

We now show that a slightly weaker version of Proposition 2(i) gives an alternative proof of (4.1.9) of [1].

Corollary 2. Let $e$ denote the exponent of $M(G), \alpha$ be a cocycle of $G$ with $o([\alpha])=e$, and $L$ be a subgroup of $G$ such that $\left[\alpha_{L}\right]=[1]$; then $e \mid[G: L]$. In particular, e divides the index of each cyclic subgroup of $G$.

Proof. By Lemma 1(i) and Proposition 2(i) we have $e|c(G, \alpha)|[G: L]$.

Finally the following type of result is useful in constructing the projective representations of a given group with specified Sylow structure.

Corollary 3. Let $\alpha$ be a cocycle of $G$ with $2 \mid o([\alpha])$, and suppose that $G$ has a dihedral Sylow 2-subgroup; then $(c(G, \alpha))_{2}=2$.

Proof. Let $P \in \operatorname{Syl}_{2}(G)$. The restriction mapping from $\operatorname{Syl}_{2}(M(G))$ into $M(P)$ is a monomorphism; hence, since $P$ has a cyclic subgroup of index 2 , we have by Proposition 1 and Corollary 1 that $(c(G, \alpha))_{2}=s\left(P, \alpha_{P}\right)=2$.

\section{REFERENCES}

1. G. Karpilovsky, Projective representations of finite groups (Monographs and textbooks in pure and applied mathematics 94, Marcel Dekker, 1985).

2. A. O. Morris, Projective representations of abelian groups, J. London Math. Soc. (2) 7 (1973), 235-238.

Department of Mathematics

UNIVERSITY COLLEGe Dublin

BELFIELD

Dublin 4, Ireland 